\title{
Higher prolactin and vasoinhibin serum levels associated with incidence and progression of retinopathy of prematurity
}

\author{
Luz Consuelo Zepeda-Romero', Miguel Vazquez-Membrillo², Elva Adan-Castro², Francisco Gomez-Aguayo', \\ Jose Alfonso Gutierrez-Padilla1,3, Eusebio Angulo-Castellanos ', Juan Carlos Barrera de Leon ${ }^{3,4}$, Cesareo Gonzalez-Bernal ${ }^{4}$, \\ Manuel Alejandro Quezada-Chalita', Alonso Meza-Anguiano', Nundehui Diaz-Lezama², Gonzalo Martinez de la Escalera², \\ Jakob Triebel ${ }^{5}$ and Carmen Clapp ${ }^{2}$
}

BACKGROUND: Retinopathy of prematurity (ROP) is a potentially blinding, retinal neovascular disease. Systemic prolactin accesses the retina to regulate blood vessels. Prolactin is proangiogenic and can be cleaved to antiangiogenic vasoinhibins. We investigated whether circulating prolactin and vasoinhibins associate with incidence and progression of ROP.

METHODS: A prospective, longitudinal, case-control study covering postnatal weeks 1 to 9 measured serum prolactin, vasoinhibins, and vascular endothelial growth factor (VEGF) weekly in 90 premature infants diagnosed as ROP or control.

RESULTS: Prolactin levels were higher in ROP than in control patients before (106.2 \pm 11.3 (SEM) vs. $64.7 \pm 4.9 \mathrm{ng} / \mathrm{ml}$, postnatal week 1) and during (120.6 \pm 10 vs. $84.7 \pm 7.5 \mathrm{ng} / \mathrm{ml}$, postnatal week 5) ROP diagnosis. Prolactin, but not gestational age, birth weight, Apgar score, sepsis, or ventilation time, correlated with ROP. The relative risk (RR) of developing ROP increased if Prolactin (PRL) levels were higher than thresholds of $80 \mathrm{ng} /$ $\mathrm{ml}(\mathrm{RR}=1.55,95 \% \mathrm{Cl}: 1.06-2.28), 100 \mathrm{ng} / \mathrm{ml}(\mathrm{RR}=1.63,95 \%$ Cl: $1.14-2.34)$, or $120 \mathrm{ng} / \mathrm{ml}(\mathrm{RR}=1.95,95 \% \mathrm{Cl}: 1.41-2.68)$. Vasoinhibin levels were $39.7 \%$ higher (95\% Cl: $4.5-77.5)$ in the circulation of ROP than in control patients at postnatal week 1 and similar thereafter, whereas VEGF serum levels were always similar.

CONCLUSION: High serum prolactin and vasoinhibin levels predict and may impact ROP progression.

$\mathbf{R}^{\mathrm{c}}$ etinopathy of prematurity (ROP) is a major cause of blindness in children throughout the world (1). ROP occurs in eyes with an incompletely developed vascular system; it causes vascular proliferation, vasopermeability, and retinal detachment, resulting in loss of vision. The pathophysiology of ROP is explained by the existence of two distinct, sequential phases of vascular development: Phase I in which retinal vascular growth slows or ceases and some vessels are lost due to premature birth. Phase II in which previous vascular changes lead to retinal hypoxia which, in turn, stimulates angiogenesis, vascular leakage, and retinal detachment (2). VEGF is the main hypoxia-inducible angiogenic factor mediating retinal neovascularization in ROP (2-4), albeit other hypoxia-inducible (erythropoietin) (5) and hypoxia-unrelated (IGF-I) $(2,6)$ angiogenic factors are certainly involved.

Prolactin (PRL) stimulates or inhibits blood vessel growth, dilation, permeability, and survival. These opposite effects result from proteolytic cleavage of the parent PRL molecule, as the full-length protein promotes angiogenesis, but vasoinhibins, the PRL fragments generated by proteolytic processing, exert antiangiogenic, proapoptotic, vasoconstrictive, and antivasopermeability effects (7). The generation, secretion, and actions of PRL and vasoinhibins occur at the hypothalamus, the pituitary, and the target tissue levels, and this organization has been defined as the PRL/vasoinhibin axis (8). This axis participates in maintaining corneal avascularity (9) and normal retinal vasculature (10) and is altered in diabetic retinopathy and in pregnancy-related vascular disorders $(7,8)$. Raising circulating PRL levels leads to vasoinhibin accumulation in the retina (11), and the elevation of intraocular vasoinhibins prevents and reverses diabetes-induced excessive retinal vasopermeability in rats $(12,13)$ and angiogenesis in a mouse model of ROP (14). Also, immunosequestering ocular vasoinhibins stimulates adult rat retinal angiogenesis (10) and reduces the apoptosis of the ocular hyaloid vasculature in neonatal rats (15). High PRL levels are present in the serum and aqueous humor of stage 5 ROP patients undergoing vitrectomy at 6 mo to $3 \mathrm{y}$ of age compared to age-matched control individuals and vasoinhibins are detected in their fibrovascular membranes and subretinal fluid (15). Moreover, PRL levels are higher in the umbilical chord blood of human infants of mothers with hypertension (16) and vasoinhibin levels increase in serum, urine, and amniotic fluid of women with pre-eclampsia,

\footnotetext{
The last two authors shared equal senior-author contribution.

'Hospital Civil "Fray Antonio Alcalde", Centro Universitario de Ciencias de la Salud, Universidad de Guadalajara, Guadalajara, Mexico; ${ }^{2}$ Institute of Neurobiology, National

University of Mexico (UNAM), Querétaro, Mexico; ${ }^{3}$ Tecnológico de Monterrey, Guadalajara, Mexico; ${ }^{4}$ Unidades Medicas de Alta Especialidad (UMAE), Hospital de Pediatría Centro Médico Nacional de Occidente (CMNO), Instituto Mexicano del Seguro Social (IMSS) Guadalajara, Mexico; ${ }^{5}$ nnstitute for Clinical Chemistry, Laboratory Medicine and Transfusion Medicine, Paracelsus Medical University, Nuremberg, Germany. Correspondence: Carmen Clapp (clapp@unam.mx)

Received 15 July 2016; accepted 19 September 2016; advance online publication 4 January 2017. doi:10.1038/pr.2016.241
} 
correlating with disease severity and reduced birth weight $(17,18)$ and in women with postpartum cardiomyopathy $(19)$.

The PRL/vasoinhibin axis is surfacing as an efficient system for regulating ocular angiogenesis, which may be altered by preterm birth in association with retinal vascular dysfunction. Here, we investigate whether the circulating levels of PRL and vasoinhibins in ROP patients associate with the onset and progression of the disease.

\section{METHODS}

\section{Subjects}

The Hospital Civil Fray Antonio Alcalde internal review board approved the study, and all procedures were performed in accord with the Declaration of Helsinki. Written informed consent was obtained from the infants' parents after fully explaining the purpose and nature of all procedures used.

Infants born at a gestational age of $<35 \mathrm{wk}$ at the Hospital Civil Fray Antonio Alcalde and at the Hospital Materno Infantil "Esperanza López Mateos" in Guadalajara, Mexico between February 2012 and January 2013 were recruited for the study. Exclusion criteria were: body weight lower than $500 \mathrm{~g}$ and medication with drugs known to affect PRL secretion (metoclopramide, methyldopa, opiates, cimetidine, or postnatal steroids). One hundred eighteen infants were identified as candidates and received permission to participate. Later, 28 patients were excluded ( 15 due to medication, 7 died before ophthalmoscopic examination, 5 missed sample collection, and 1 was transferred to another hospital) leaving 90 study subjects. All infants were hospitalized in a neonatal intensive care unit and received enteral feeding with standard formula and increasing amounts of breast milk, supplemented in some cases with parenteral glucose, amino acids, and fat.

\section{Diagnosis of ROP}

ROP was diagnosed when indirect ophthalmoscopy demonstrated the presence of a structure perpendicular to and located at the tips of the developing retinal blood vessels that divide the vascular from the avascular retina. Weekly examinations began at $3 \mathrm{wk}$ postnatal age corresponding to $\geq 31$ wk of postmenstrual age (sum of gestational age and postnatal age).

\section{Severity of ROP}

The severity scale established by the International Classification of Retinopathy of Prematurity (20) was used. Briefly, a three-level stage was assigned with 0 denoting no disease, 1 and 2 for mild disease (demarcation line and ridge, respectively), and 3 for severe disease (ridge with extraretinal fibrovascular proliferation). No infant developed ROP stages 4 or 5 .

\section{Sample Collection, PRL and Vasoinhibin Analyses}

Serum samples $(0.5 \mathrm{ml})$ taken weekly were stored at $-80^{\circ} \mathrm{C}$ until assayed. PRL levels were quantified using the IMMULITE $2000 \mathrm{XPi}$ immunoassay system (Siemens, Munich, Germany). The intra-assay and inter-assay coefficients of variance were less than $1 \%$. For vasoinhibin determination, $50 \mu \mathrm{l}$ of serum was immunoprecipitated with $2 \mu \mathrm{l}$ of antihuman PRL antiserum (HC1) obtained and characterized as reported $(15,17)$ using the previously published technique (17) with modifications. Briefly, samples were incubated with $\mathrm{HCl}$ antiserum overnight followed by a 2 -h incubation with protein-A Sepharose beads $(35 \mu \mathrm{l}$, Sigma Aldrich, St Louis, MO). The samples were then centrifuged and washed with NET-gel $(0.05 \mathrm{M}$ Tris, $0.15 \mathrm{M} \mathrm{NaCl}, 5 \mathrm{mMol} / \mathrm{l}$ ethylenediaminetetraacetic acid, $0.1 \%$ Nonidet P- $40,0.25 \%$ gelatin from bovine skin, $0.02 \%$ sodium azide, $\mathrm{pH} 8$ ), with NET-gel containing $10 \%$ SDS, and finally with $0.05 \mathrm{M}$ Tris, $1 \%$ Nonidet P- $40, \mathrm{pH} 8$. Each wash was for $10 \mathrm{~min}$ at $4{ }^{\circ} \mathrm{C}$ under gentle agitation, and centrifugation was at $9,000 \times$ $\mathrm{g}$ for $5 \mathrm{~min}$. Final pellets were subjected to sodium dodecyl sulfate polyacrilamide gel electrophoresis on a 15\% acrylamide gel under reducing conditions, and blotted onto nitrocellulose membranes. Membranes were blocked with $4 \%$ skim milk in $0.1 \%$ Tween-20-PBS for $1 \mathrm{~h}$ at room temperature and probed overnight at $4{ }^{\circ} \mathrm{C}$ with $4 \mu \mathrm{g} / \mathrm{ml}$ anti-human PRL monoclonal antibodies (mAb5602, Diagnostic Biochem Canada Inc., London, Canada) reacting with the N-terminus of PRL (21), which defines vasoinhibins $(7,8)$. Detection used alkaline phosphatase-coupled donkey anti-mouse secondary antibodies (Santa Cruz Biotechnology, Santa Cruz, CA) and a colorimetric detection kit (Bio-Rad Laboratories, Hercules, CA). Optical density values were determined with Quantity One image analysis software (Bio-Rad). Serum VEGF levels were quantified with an ELISA (Enzyme-Linked ImmunoSorbent Assay) (Thermo Fisher Scientific, Waltham, MA) that recognizes both natural and recombinant human VEGF-165 with intra- and inter-assay coefficients of variance of $<4.7$ and $<8.1 \%$, respectively.

\section{Statistical Analysis}

The Sigma Stat 7.0 software (Systat Software, San Jose, CA) was used. The $t$-test evaluated differences between two groups when their distribution was normal and the Mann-Whitney $U$-test when it was not. The chi-square test was used to test the association between categorical variables. The Pearson correlation test evaluated the level of association between numerical variables. The Kruskal-Wallis test was employed as a global test to examine longitudinal differences among three groups. Differences between three groups were determined by one-way ANOVA followed by Bonferroni's post hoc comparison test. Contingency tables analyzed with Fisher's exact test determined relative risks. Multivariate logistic regression analysis was used to evaluate the association of several risk variables with ROP using MedCalc software version 16 . The threshold for significance was set at $P<0.05$.

\section{RESULTS}

Table 1 shows the demographics and clinical characteristics of the patients studied. ROP was diagnosed between postnatal weeks 3 and 9 ( $5 \pm 1.5$ (SEM) weeks, 95\% CI: 4.6-5.5) and occurred in 49 (54.4\%) of the 90 infants in the study. Of these, 27 (55.1\%) had mild ROP (14 stage 1 and 13 stage 2) and 22 (44.9\%) had severe ROP (stage 3 ). The control and ROP groups were similar in gestational age, gender, Apgar score (5 min after birth), and ventilation time, but different in birth weight (Table 1). A nonsignificant higher incidence of sepsis occurred in infants who developed ROP (Table 1).

PRL circulating levels in the first postnatal week were significantly higher in the group that later developed ROP than

Table 1. Demographic and clinical characteristics of the population in the study

\begin{tabular}{|c|c|c|c|c|c|}
\hline & $\begin{array}{l}\text { Control } \\
(n=41)\end{array}$ & $\begin{array}{c}95 \% \\
\mathrm{Cl}\end{array}$ & $\begin{array}{c}\text { ROP } \\
(n=49)\end{array}$ & $\begin{array}{c}95 \% \\
\mathrm{Cl}\end{array}$ & $P$ \\
\hline $\begin{array}{l}\text { Gestational age } \\
\text { (weeks) }\end{array}$ & $30.8 \pm 0.3$ & $30.2-31.5$ & $30.2 \pm 0.3$ & $29.7-30.8$ & $0.16^{\mathrm{a}}$ \\
\hline Gender F n (\%) & $20(48.7)$ & & $26(53.1)$ & & $0.83^{b}$ \\
\hline Apgar (5 min) & $8.28 \pm 0.2$ & 7.9-8.7 & $8.22 \pm 0.1$ & $8.0-8.5$ & $0.77^{c}$ \\
\hline $\begin{array}{l}\text { Ventilation time } \\
\text { (days) }\end{array}$ & $3.53 \pm 0.9$ & $1.6-5.4$ & $6.54 \pm 1.8$ & $2.7-10.3$ & $0.22^{c}$ \\
\hline Birth weight (g) & $1,317 \pm 34.9$ & $1,247-1,388$ & $1,193 \pm 35.8$ & $1,121-1,265$ & $0.01^{c}$ \\
\hline Sepsis n (\%) & $29(70.7)$ & & $42(85.7)$ & & $0.11^{\mathrm{b}}$ \\
\hline $\begin{array}{l}\text { ROP diagnosis } \\
\text { (weeks) }\end{array}$ & $30.8 \pm 0.3$ & $26-33$ & $30.2 \pm 0.3$ & $26-34$ & 0.13 \\
\hline $\begin{array}{l}\text { (Postnatal } \\
\text { weeks) }\end{array}$ & & & $5 \pm 1.5$ & $4.6-5.5$ & \\
\hline Mild ROP (\%) & & & $27(55.1)$ & & \\
\hline Severe ROP (\%) & & & $22(44.9)$ & & \\
\hline
\end{tabular}

Mild ROP corresponds to disease stages 1 and 2, severe ROP to disease stage 3. Values are means \pm SEM.

$P$ values compare control and ROP groups and are from $t$-test ${ }^{\mathrm{a}}, \chi^{2}$ test ${ }^{\mathrm{b}}$, and MannWhitney $U$-test ${ }^{c}$.

$\mathrm{Cl}$, confidence intervals; $\mathrm{ROP}$, retinopathy of prematurity. 
in the control group $(106.2 \pm 11.2$ (SEM) vs. $64.7 \pm 4.8 \mathrm{ng} / \mathrm{ml}, P$ $=0.001$ ). Although lower birth weight and higher PRL values were associated with ROP, there was no association between birth weight and PRL levels when considering all patients (control and ROP) $(r=0.05, P=0.63$; Figure 1a), suggesting that reduced birth weight and high serum PRL levels are not causally related, albeit only correlation was examined. In addition, serum PRL values were similar between patients with or without sepsis $(P=0.66$; Figure $1 \mathbf{b})$, in spite of the tendency of sepsis to associate with ROP.

PRL levels at postnatal week 1 correlated with increased relative risk of developing ROP (Table 2). Comparing the bottom vs. the top PRL quartile thresholds, the relative risk increased from 1.3 to 1.95 , with an increment in the relative risk between each threshold. At the highest PRL levels, the risk of ROP development increased twofold (Table 2).

Next, we investigated whether or not the predictive value of systemic PRL during the first week of life was associated with the predictive value of known risk variables for ROP, i.e., low birth weight, low gestational age, low Apgar score, sepsis, and long ventilation support $(2,22)$. Accordingly, a multivariate logistic regression analysis was performed, in which PRL levels at postnatal week 1, were entered together with the above confounding risk factors (Table 3 ). The analysis demonstrated that elevated PRL levels at postnatal week 1 correlated significantly $(P=0.013)$ with the occurrence of ROP, whereas all other independent variables did not (Table 3 ), suggesting that high systemic PRL by itself is a risk factor for ROP.

PRL circulating levels were higher in the ROP group not only during postnatal week 1 , but also during postnatal weeks 2 and 4 to 7 (Figure 1c). Likewise, PRL levels were higher at weeks 30 and 32 to 35 of postmenstrual age in infants with ROP (Figure 1d). These findings suggest that time after birth, more than infant development, influences the changes of circulating PRL in ROP. Since ROP was diagnosed around postnatal week 5 (Table 1), i.e., around $35 \mathrm{wk}$ postmenstrual age, increased circulating PRL levels preceded and coexisted with the onset of ROP.

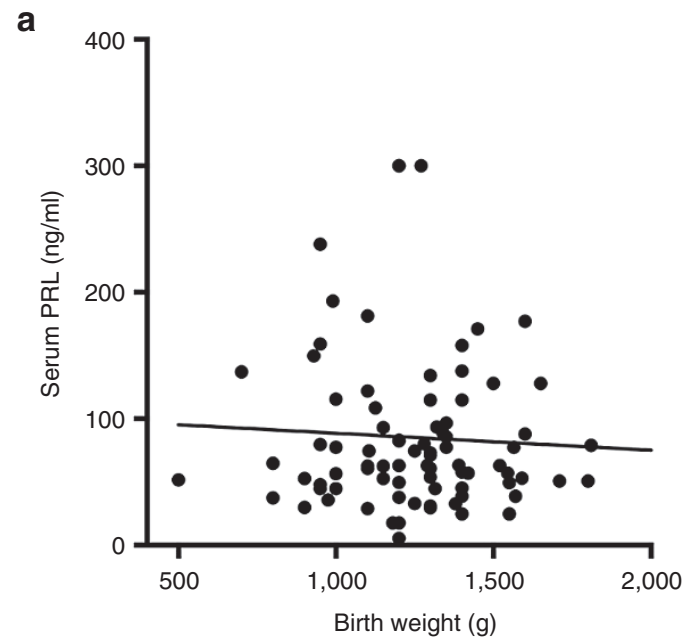

C

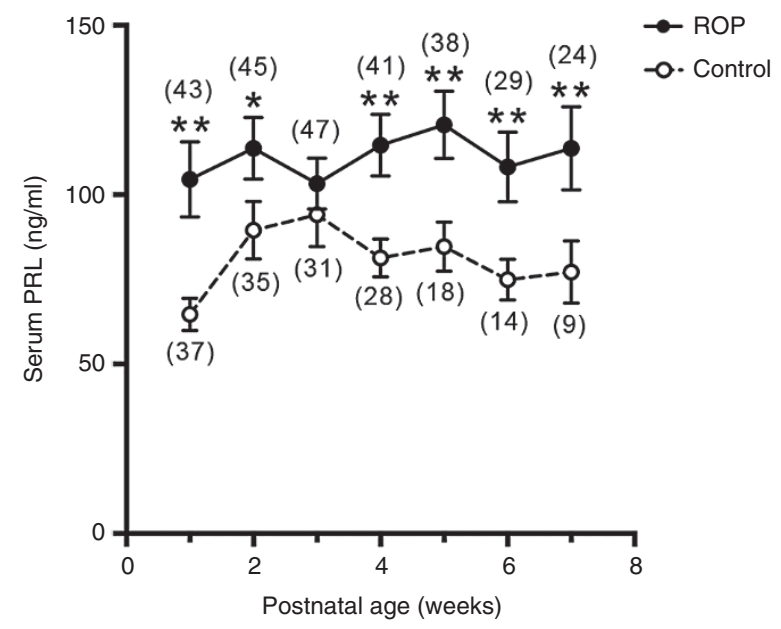

b

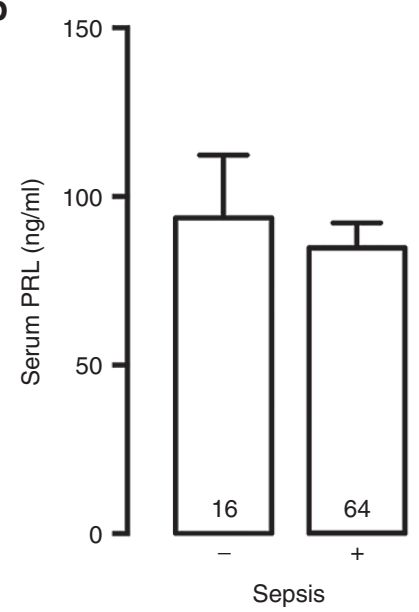

d

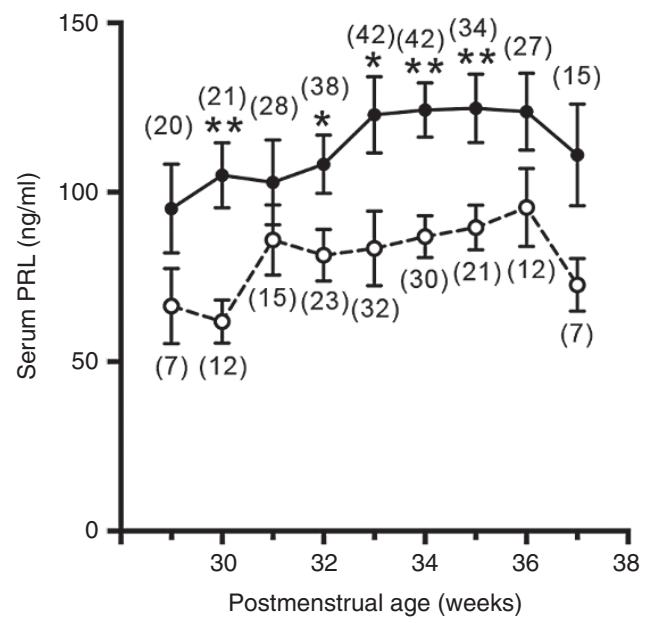

Figure 1. Higher Prolactin (PRL) serum levels associate with incidence and progression of retinopathy of prematurity (ROP). (a) There is no correlation $(r=0.05, P=0.6)$ between PRL serum levels at postnatal week 1 and birth weight in the study population. (b) Serum PRL levels at postnatal week 1 are similar $(P=0.66)$ between patients with or without sepsis. Values are means \pm SEM. Number of patients $(n)$ is indicated inside bars. Serum PRL levels are higher throughout postnatal (c) and postmenstrual (d) age in ROP patients (black circles) compared with non-ROP patients (white circles). Values are means \pm SEM. Number of patients $(n)$ is indicated inside parentheses. ${ }^{*} P<0.04$ and ${ }^{* *} P<0.005$ vs. non-ROP patients (t-test). 


\section{Articles $\mid$ zepeda-Romeroetal.}

The longitudinal values of serum PRL at different levels of ROP status (control, mild, and severe) are shown in Figure 2. The Kruskal-Wallis test yielded $P$ values of 0.0001 and 0.0002 against postnatal age and postmenstrual age, respectively, indicating significant longitudinal differences in "mean PRL levels" between the three groups. One-way ANOVA, applying the Bonferroni correction, showed significant differences between mild-ROP and control groups at postnatal weeks 1

Table 2. PRL serum levels and relative risk of ROP

\begin{tabular}{lccc}
\hline $\begin{array}{l}\text { Serum PRL }(\mathrm{ng} / \mathrm{ml}) \\
\text { at postnatal week } 1\end{array}$ & $\begin{array}{c}\text { Relative } \\
\text { risk }\end{array}$ & $95 \% \mathrm{Cl}$ & $P$ \\
\hline$>60$ & 1.33 & $0.86-2.06$ & 0.256 \\
$>80$ & 1.55 & $1.06-2.28$ & 0.038 \\
$>100$ & 1.63 & $1.14-2.34$ & 0.023 \\
$>120$ & 1.95 & $1.41-2.68$ & 0.002 \\
\hline
\end{tabular}

$P$ values are form contingency tables analyzed with Fisher's exact test.

PRL, Prolactin; ROP, retinopathy of prematurity.

Table 3. Multivariate analysis showing the relationship of independent variables with the risk of ROP in infants with ROP and controls

\begin{tabular}{lccc}
\hline Independent variable & OR & $95 \% \mathrm{Cl}$ & $P$ \\
\hline Gestational age & 0.926 & $0.624-1.375$ & 0.704 \\
Apgar (5 min) & 2.209 & $0.954-5.117$ & 0.064 \\
Ventilation time & 1.037 & $0.890-1.209$ & 0.640 \\
Birth weight & 0.999 & $0.996-1.003$ & 0.675 \\
Sepsis & 0.555 & $0.150-2.060$ & 0.379 \\
PRL & 1.016 & $1.003-1.029$ & 0.013 \\
\hline
\end{tabular}

$P$ values are from multivariate conditional logistic regression analysis. $\mathrm{Cl}$, confidence interval; PRL, Prolactin; ROP, retinopathy of prematurity. and 4 , while severe-ROP and control groups were different at postnatal weeks 1, 4, 5, and 6 (Figure 2a). Likewise, differences between mild-ROP and control were significant only at postmenstrual week 30 , whereas severe-ROP and control were significantly different at postnatal weeks 32, 33, 34, and 35 (Figure 2b). The fact that serum PRL was significantly higher for a longer time in severe ROP than in mild ROP suggests that PRL levels increase as the disease worsens.

Next, we measured vasoinhibin levels in the circulation of control and ROP patients. Serum samples from control and ROP patients were immunoprecipitated for vasoinhibin detection at postnatal weeks $1,2,4$, and 6; representative Western blots are shown in Figure 3. As expected (17), the light chain of immunoglobulin molecules, which has a mass of approximately $25 \mathrm{kDa}$ interfered with the detection of full-length $23 \mathrm{kDa}$ PRL. Serum contained immunoreactive proteins of 14 and $16 \mathrm{kDa}$ that may correspond to vasoinhibins, which range in size from 11 to $18 \mathrm{kDa}$ and contain the $\mathrm{N}$-terminal end of PRL (8). The combined densitometric value of the two vasoinhibins measured $1 \mathrm{wk}$ after birth was significantly higher $(P=0.03)$ in infants who later developed ROP compared to control patients (Figure 3a). In contrast, concentrations of the two vasoinhibins were similar between the control and the ROP groups at postnatal weeks $2(P=0.08), 4(P=0.84)$, and $6(P=0.83)$ (Figure $3 b, c)$. These findings suggest that systemic vasoinhibin levels increase during the first week of life in association with the later development of ROP.

VEGF is present in the circulation of neonates (23), increases in the vitreous of ROP patients (24), and plays a central role in the development and progression of ROP (2-4). However, in this study, the control and ROP groups had similar circulating levels of VEGF throughout the 6 wk of postnatal age (Figure 4). a

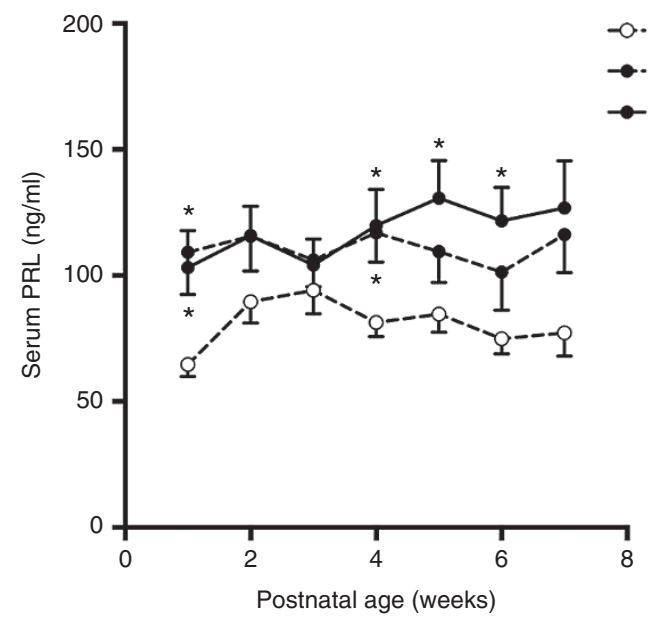

b

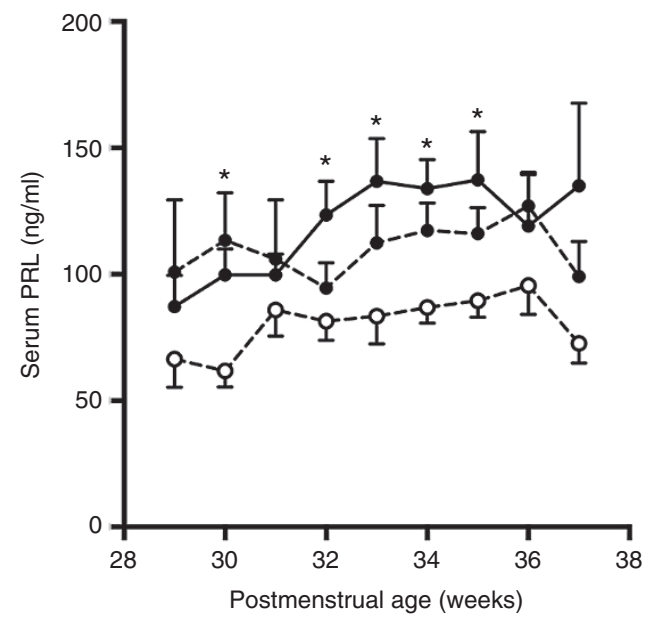

Figure 2. Prolactin (PRL) levels remain higher for longer times in severe vs. mild retinopathy of prematurity (ROP). Serum PRL levels throughout postnatal (a) and postmenstrual (b) age in ROP patients compared with non-ROP patients as a function of disease severity. Black circles and continuous line indicate PRL values in severe ROP; black circles and discontinuous line indicate PRL values in mild ROP; and white circles and discontinuous line indicate non-ROP PRL values. Severe ROP corresponds to disease stage 3 and mild ROP corresponds to disease stages 1 and 2. Values are means \pm SEM. Number of patients for severe, mild, and non-ROP at different postnatal age, are: week $1(21,22,37)$, week $2(22,23,35)$, week $3(21,26,31)$, week $4(19,22,28)$, week 5 $(20,18,18)$, week $6(14,15,14)$, and week $7(9,12,12)$. Number of patients for severe, mild, and non-ROP at different postmenstrual age, are: week $29(13,7,7)$, week $30(13,8,12)$, week $31(14,14,15)$, week $32(18,20,23)$, week $33(18,24,32)$, week $34(18,24,30)$, and week $35(14,20,21)$, week $36(11,16,12)$, and week 37 $(5,10,7) .{ }^{*} P<0.04$ vs. non-ROP (one-way ANOVA and Bonferroni's post hoc comparison test). 
a

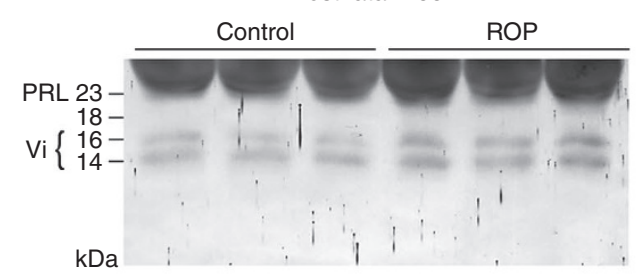

C

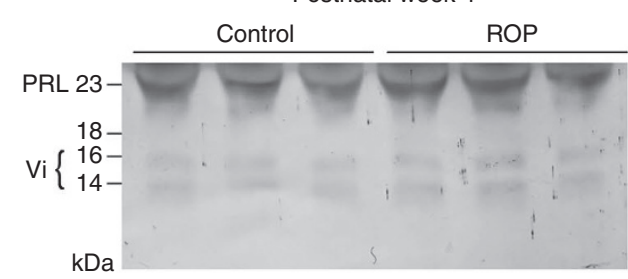

b

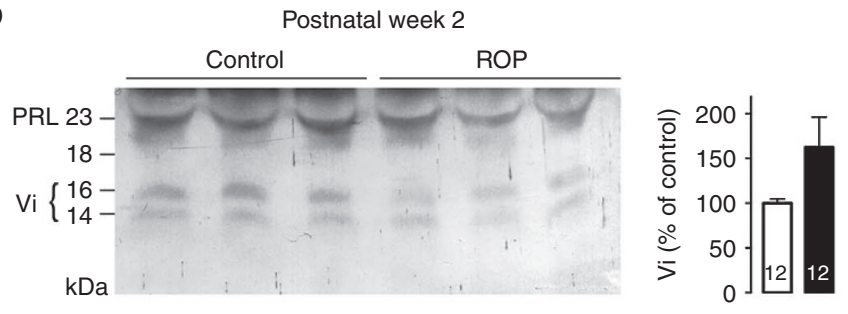

d

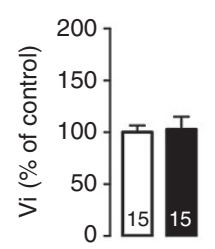

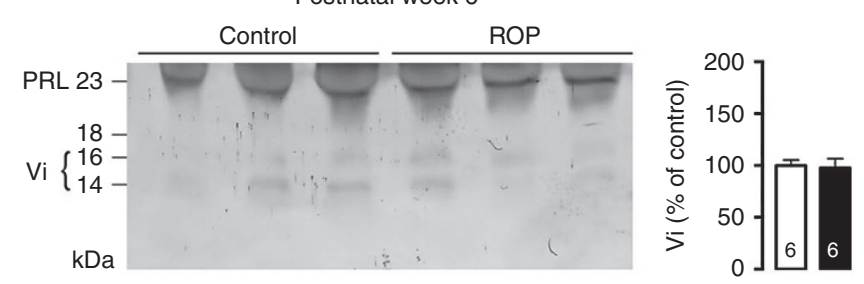

Figure 3. Vasoinhibins, serum levels are elevated during postnatal week 1 in retinopathy of prematurity (ROP) patients. Representative Western blots evaluating vasoinhibins (Vi) in serum samples from three non-ROP (control) and three ROP patients at postnatal weeks 1 (a), 2 (b)), 4 (c), and 6 (d). Before Western blot analysis, serum samples were immunoprecipitated with an anti-PRL antiserum and all blots were probed with an anti-Prolactin (PRL) mAb that reacts with the N-terminal end of PRL. Numbers on the left side indicate the position of full-length PRL ( $23 \mathrm{kDa})$ and of vasoinhibins of 18,16 , and $14 \mathrm{kDa}$. Bar graphs on the right show the combined densitometric values (means $\pm \mathrm{SEM}$ ) of the two vasoinhibin isoforms (16 and $14 \mathrm{kDa})$ detected in patients who developed ROP, expressed as a percentage of those in the control group. Number of patients is indicated inside bars. ${ }^{*} P<0.03$ vs. control ( $t$-test).

\section{DISCUSSION}

This study evaluated the serum levels of the proangiogenic hormone PRL and its antiangiogenic derivatives (vasoinhibins) before and during ROP. We report a significant increase of PRL both before and during ROP and of vasoinhibins only before ROP, indicating a dysregulation of the PRL/vasoinhibin axis that has a time course consistent with having a role in promoting the early (vasoinhibitory) and the late (vasoproliferative) phases of the disease. We suggest that PRL and vasoinhibin serum measurements during the first week of life can help predict ROP, and that their circulating levels could directly impact onset and progression of this disease due to their opposing effects on ocular blood vessels.

During pregnancy, PRL rises progressively in maternal and fetal blood and reaches peak values (around $200 \mathrm{ng} / \mathrm{ml}$ ) at term $(25,26)$. In addition to circulating PRL, the decidua produces PRL that accumulates in the amniotic fluid to levels 10 to 100 times higher than those of maternal or fetal blood (27). The human placenta cleaves PRL to vasoinhibins (17), and vasoinhibins are present both in maternal circulation and amniotic fluid at the end of gestation (17). Premature infants have lower PRL circulating levels than full-term infants at birth, but this difference disappears between postnatal day 4 and postnatal week 6 when PRL values decline in full-term, but not in premature infants (28).

Here, we confirmed the sustained prolactinemia reported throughout the first $6 \mathrm{wk}$ of life in premature infants and showed that PRL levels are higher in premature patients who develop ROP than in the control group. Gender, gestational age, Apgar score, sepsis, and ventilation time do not appear to correlate with PRL levels, as these parameters were similar between the ROP and the control groups. Also, serum PRL

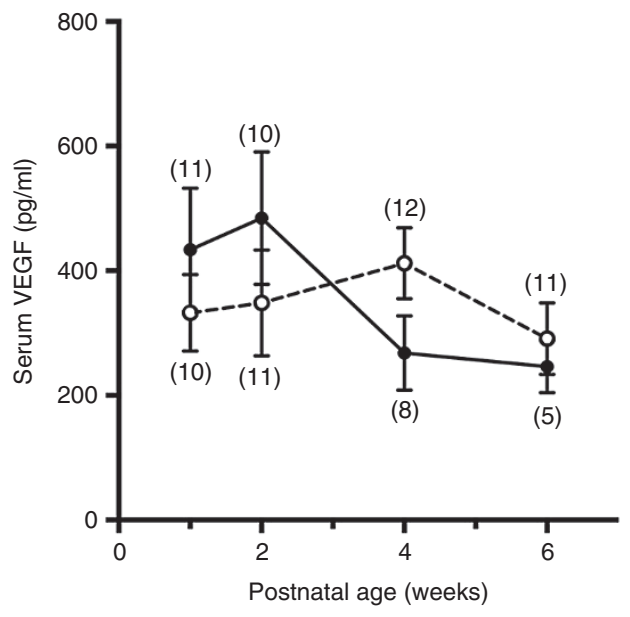

Figure 4. VEGF serum levels do not change in retinopathy of prematurity (ROP) patients. VEGF serum levels (means \pm SEM) as evaluated by ELISA in non-ROP (white circles) and ROP (black circles) patients at postnatal weeks $1,2,4$, and 6 . Number of patients is indicated inside parenthesis.

levels during the first week of life did not correlate with birth weight or with incidence of sepsis, suggesting that higher PRL levels in infants who will develop ROP are not associated with fetal growth and that sepsis-related stress does not stimulate the secretion of PRL by the premature pituitary gland. Lack of correlation between serum PRL and birth weight, sex, and Apgar score is not unexpected (16), but lower PRL levels have been associated with a need for increased ventilatory assistance in premature neonates (29). In this context, gestational age, birth weight, sepsis, Apgar score, and duration of ventilation are known risk factors for $\operatorname{ROP}(2,22)$, which may obscure an association of PRL with ROP outcome. However, adjusting for these confounding factors through multivariate 
logistic regression analysis showed that PRL levels during the first week of life were the only independent variable significantly associated with ROP. Also, the pattern of relative risk across PRL quartile categories was linear; statistical differences rose with each increment in PRL threshold values, and the top quartile had a twofold higher risk of developing ROP. These analyses suggest that PRL itself is a predictive risk variable for ROP.

Higher systemic levels of PRL may impact disease onset and progression. PRL serum levels were significantly higher in ROP than control patients during the first (vasoinhibitory) and the second (vasoproliferative) phases of ROP, i.e., at postmenstrual weeks 30 and 32 to 35 , respectively. Furthermore, PRL levels remained higher for longer times in severe vs. mild ROP, implying that PRL levels increase as the disease worsens. Because PRL can inhibit or stimulate the proliferation, dilation, permeability, and regression of blood vessels, its higher levels in ROP patients may help drive both the vasoinhibitory and the vasoproliferative phases of ROP. The paradox of opposite effects is explained by the PRL/ vasoinhibin axis. PRL is proteolytically converted to vasoinhibins in both the pituitary gland and the target tissue, and PRL and vasoinhibins activate distinct receptor complexes and signaling pathways to stimulate and inhibit the growth and survival of blood vessels, respectively (for reviews, see refs. $(7,8))$.

Vasoinhibins were detected in all serum samples from premature infants, perhaps because the high levels of circulating PRL during the neonatal period favor their generation. However, in spite of PRL levels being higher throughout ROP, vasoinhibins were significantly elevated only during the vasoinhibitory phase of the disease. This suggests that enhanced PRL cleavage occurs early in ROP and that the resulting vasoinhibins may contribute to the cessation and regression of normal vascular development that initiate the disease. Immune sequestration of vasoinhibins in neonatal rats reduces the apoptosis-mediated regression of intraocular hyaloid vessels (15), and gene transfer of vasoinhibins via an adenoviral vector inhibits angiogenesis in a mouse model of ROP (14). Moreover, expression of matrix metalloproteases (MMP) 2 and 9 is enhanced in eyes of mice with experimental ROP before the vasoproliferative phase $(30,31)$, and several MMP, including MMP-2 and MMP-9, cleave PRL to vasoinhibins (32). Cathepsin D, which also generates vasoinhibins (21), is also present in various eye compartments (33) and is downregulated in the circulation of patients with diabetic retinopathy (34). The fact that vasoinhibin levels are not elevated during the vasoproliferative phase of ROP suggests that PRL-cleaving activity becomes downregulated in this second phase which, coupled to higher PRL levels, may favor the proangiogenic outcome of PRL action.

This study has several limitations. The ROP and control populations were small and similar in terms of demographic and clinical characteristics, possibly masking associations between PRL levels and known ROP risk variables. While relative risk associations are robust, the analysis using specific quartile cut points precludes relating absolute PRL concentrations to risk. Also, the reduced number of patients after staging ROP, together with the lack of patients having a more severe disease (stages 4 and 5), limits the statistical power to less than that required for definitive statements regarding disease progression. Another important limitation of our study is the absence of a quantitative assay for determining vasoinhibins. The chemioluminescent immunometric assay employed here for evaluating PRL essentially measures PRL and not vasoinhibins, as vasoinhibins have low $(<10 \%)$ immunoreactivity in this assay (data not shown) and in other quantitative PRL immunoassays (35). In the absence of a quantitative assay for vasoinhibins, their serum levels can only be determined semi-quantitatively by immunoprecipitation followed by Western blotting and optical densitometry. This methodology revealed vasoinhibins of 14 and $16 \mathrm{kDa}$, the masses of the more widely characterized vasoinhibin isoforms $(7,8)$. However small, albeit significant, differences in vasoinhibin serum levels could have escaped detection by this technique. Finally, while the incorporation of systemic PRL into the eye $(11,36)$ and its effects on the vasculature (10-14) and neural tissue (37) of the retina are well established, there is insufficient information concerning the mechanisms mediating the systemic and intraocular conversion of PRL to vasoinhibins in the neonate. Studies addressing these aspects should help substantiate the role of systemic PRL and vasoinhibins as factors impacting ROP.

In conclusion, our work supports the value of measuring PRL and vasoinhibin serum levels to predict ROP and suggests they contribute to the pathophysiology of the disease. VEGF is the major driver of ROP in the retina, and its intraocular levels are indicative of pathologic state of ROP $(3,4)$; however, the serum concentration of VEGF has little $(38)$ or no $(39,40)$ (present findings) predictive value for ROP, highlighting the utility of circulating PRL and vasoinhibins as predictive markers.

\section{ACKNOWLEDGMENTS}

We thank Fernando López-Barrera, and Gabriel Nava, for their technical assistance, Fernanda Parada-Dávila, for statistic support, and Dorothy D. Pless, for critically editing the manuscript.

\section{STATEMENT OF FINANCIAL SUPPORT}

This study was supported by the National Council of Science and Technology of Mexico (CONACYT), grant SALUD-2012-01-179506 to C.C. M.V-M. and N.D-L. are doctoral students from Programa de Doctorado en Ciencias Biomédicas, Universidad Nacional Autónoma de México (UNAM) and received fellowships 289715 (MVM) and 245224 (NDL) from the CONACYT. E.A-C. is a master's degree student from Programa de Maestría en Ciencias (Neurobiología), UNAM and received fellowship 588869 from CONACYT.

Disclosure: All authors declare no conflict of interest.

\section{REFERENCES}

1. Friddle K. Pathogenesis of retinopathy of prematurity: Does inflamation play a role? Newborn Infant Nurs Rev 2013; 13:161-165.

2. Hellström A, Smith LE, Dammann O. Retinopathy of prematurity. Lancet 2013;382:1445-57.

3. Budd SJ, Hartnett ME. Increased angiogenic factors associated with peripheral avascular retina and intravitreous neovascularization: a model of retinopathy of prematurity. Arch Ophthalmol 2010;128:589-95.

4. Harder BC, von Baltz S, Jonas JB, Schlichtenbrede FC. Intravitreal lowdosage bevacizumab for retinopathy of prematurity. Acta Ophthalmol 2014;92:577-81. 
5. Sato T, Kusaka S, Shimojo H, Fujikado T. Vitreous levels of erythropoietin and vascular endothelial growth factor in eyes with retinopathy of prematurity. Ophthalmology 2009;116:1599-603.

6. Hellström A, Engström E, Hård AL, et al. Postnatal serum insulin-like growth factor I deficiency is associated with retinopathy of prematurity and other complications of premature birth. Pediatrics 2003;112:1016-20.

7. Clapp C, Thebault S, Macotela Y, Moreno-Carranza B, Triebel J, Martínez de la Escalera G. Regulation of blood vessels by prolactin and vasoinhibins. Adv Exp Med Biol 2015;846:83-95.

8. Triebel J, Bertsch T, Bollheimer C, et al. Principles of the prolactin/vasoinhibin axis. Am J Physiol Regul Integr Comp Physiol 2015;309:R1193-203.

9. Dueñas Z, Torner L, Corbacho AM, et al. Inhibition of rat corneal angiogenesis by $16-\mathrm{kDa}$ prolactin and by endogenous prolactin-like molecules. Invest Ophthalmol Vis Sci 1999;40:2498-505.

10. Aranda J, Rivera JC, Jeziorski MC, et al. Prolactins are natural inhibitors of angiogenesis in the retina. Invest Ophthalmol Vis Sci 2005;46:2947-53.

11. Arnold E, Rivera JC, Thebault S, et al. High levels of serum prolactin protect against diabetic retinopathy by increasing ocular vasoinhibins. Diabetes 2010;59:3192-7.

12. García C, Aranda J, Arnold E, et al. Vasoinhibins prevent retinal vasopermeability associated with diabetic retinopathy in rats via protein phosphatase 2A-dependent eNOS inactivation. J Clin Invest 2008;118:2291-300.

13. Ramírez M, Wu Z, Moreno-Carranza B, et al. Vasoinhibin gene transfer by adenoassociated virus type 2 protects against VEGF- and diabetes-induced retinal vasopermeability. Invest Ophthalmol Vis Sci 2011;52:8944-50.

14. Pan $H$, Nguyen NQ, Yoshida $H$, et al. Molecular targeting of antiangiogenic factor $16 \mathrm{~K}$ hPRL inhibits oxygen-induced retinopathy in mice. Invest Ophthalmol Vis Sci 2004;45:2413-9.

15. Dueñas Z, Rivera JC, Quiróz-Mercado H, et al. Prolactin in eyes of patients with retinopathy of prematurity: implications for vascular regression. Invest Ophthalmol Vis Sci 2004;45:2049-55.

16. Parker CR Jr, MacDonald PC, Guzick DS, Porter JC, Rosenfeld CR, Hauth JC. Prolactin levels in umbilical cord blood of human infants: relation to gestational age, maternal complications, and neonatal lung function. Am J Obstet Gynecol 1989;161:795-802.

17. González C, Parra A, Ramírez-Peredo J, et al. Elevated vasoinhibins may contribute to endothelial cell dysfunction and low birth weight in preeclampsia. Lab Invest 2007;87:1009-17.

18. Leaños-Miranda A, Márquez-Acosta J, Cárdenas-Mondragón GM, et al. Urinary prolactin as a reliable marker for preeclampsia, its severity, and the occurrence of adverse pregnancy outcomes. J Clin Endocrinol Metab 2008;93:2492-9.

19. Hilfiker-Kleiner D, Kaminski K, Podewski E, et al. A cathepsin D-cleaved $16 \mathrm{kDa}$ form of prolactin mediates postpartum cardiomyopathy. Cell 2007;128:589-600.

20. International Committee for the Classification of Retinopathy of P. The international classification of retinopathy of prematurity revisited. Arch Ophthalmol 2005; 123:991-999.

21. Piwnica D, Touraine P, Struman I, et al. Cathepsin D processes human prolactin into multiple 16K-like $\mathrm{N}$-terminal fragments: study of their antiangiogenic properties and physiological relevance. Mol Endocrinol 2004;18:2522-42.
22. Flynn JT. Acute proliferative retrolental fibroplasia: multivariate risk analysis. Trans Am Ophthalmol Soc 1983;81:549-91.

23. Hellgren G, Löfqvist C, Hård AL, et al. Serum concentrations of vascular endothelial growth factor in relation to retinopathy of prematurity. Pediatr Res 2016;79:70-5.

24. Velez-Montoya R, Clapp C, Rivera JC, et al. Intraocular and systemic levels of vascular endothelial growth factor in advanced cases of retinopathy of prematurity. Clin Ophthalmol 2010;4:947-53.

25. Tyson JE, Hwang P, Guyda H, Friesen HG. Studies of prolactin secretion in human pregnancy. Am J Obstet Gynecol 1972;113:14-20.

26. Winters AJ, Colston C, MacDonald PC, Porter JC. Fetal plasma prolactin levels. J Clin Endocrinol Metab 1975;41:626-9.

27. Ben-Jonathan N, Munsick RA. Dopamine and prolactin in human pregnancy. J Clin Endocrinol Metab 1980;51:1019-25.

28. Guyda HJ, Friesen HG. Serum prolactin levels in humans from birth to adult life. Pediatr Res 1973;7:534-40.

29. Lucas A, Baker BA, Cole TJ. Plasma prolactin and clinical outcome in preterm infants. Arch Dis Child 1990;65:977-83.

30. Notari L, Miller A, Martínez A, et al. Pigment epithelium-derived factor is a substrate for matrix metalloproteinase type 2 and type 9: implications for downregulation in hypoxia. Invest Ophthalmol Vis Sci 2005;46: 2736-47.

31. Zhang W, Yokota $\mathrm{H}, \mathrm{Xu} \mathrm{Z}$, et al. Hyperoxia therapy of pre-proliferative ischemic retinopathy in a mouse model. Invest Ophthalmol Vis Sci 2011;52:6384-95.

32. Macotela Y, Aguilar MB, Guzmán-Morales J, et al. Matrix metalloproteases from chondrocytes generate an antiangiogenic $16 \mathrm{kDa}$ prolactin. J Cell Sci 2006;119(Pt 9):1790-800.

33. Hayasaka S. Lysosomal enzymes in ocular tissues and diseases. Surv Ophthalmol 1983;27:245-58.

34. Chen YH, Chou HC, Lin ST, Chen YW, Lo YW, Chan HL. Effect of high glucose on secreted proteome in cultured retinal pigmented epithelium cells: its possible relevance to clinical diabetic retinopathy. J Proteomics 2012;77:111-28.

35. Clapp C, Sears PS, Russell DH, Richards J, Levay-Young BK, Nicoll CS. Biological and immunological characterization of cleaved and $16 \mathrm{~K}$ forms of rat prolactin. Endocrinology 1988;122:2892-8.

36. O'Steen WK, Sundberg DK. Patterns of radioactivity in the eyes of rats after injection of iodinated prolactin. Ophthalmic Res 1982;14:54-62.

37. Arnold E, Thebault S, Baeza-Cruz G, et al. The hormone prolactin is a novel, endogenous trophic factor able to regulate reactive glia and to limit retinal degeneration. J Neurosci 2014;34:1868-78.

38. Kong L, Bhatt AR, Demny AB, et al. Pharmacokinetics of bevacizumab and its effects on serum VEGF and IGF-1 in infants with retinopathy of prematurity. Invest Ophthalmol Vis Sci 2015;56:956-61.

39. Pieh C, Agostini H, Buschbeck C, et al. VEGF-A, VEGFR-1, VEGFR-2 and Tie2 levels in plasma of premature infants: relationship to retinopathy of prematurity. Br J Ophthalmol 2008;92:689-93.

40. Woo SJ, Park KH, Lee SY, et al. The relationship between cord blood cytokine levels and perinatal factors and retinopathy of prematurity: a gestational age-matched case-control study. Invest Ophthalmol Vis Sci 2013;54:3434-9. 